\title{
Quantitation of Enamel Demineralization Mechanisms: III. A Critical Examination of the Hydroxyapatite Model
}

\author{
WILLIAM I. HIGUCHI, NASIM A. MIR, PRAFULL R. PATEL, JOHN W. BECKER, \\ and JOHN J. HEFFERREN \\ College of Pharmacy, University of Michigan, Ann Arbor, Michigan 48104, and American \\ Dental Association, Chicago, Illinois 60611
}

A physical model which assumes that hydroxyapatite is the thermodynamic governing phase for the acid dissolution-rate behavior of enamel has been critically examined theoretically and experimentally. Both enamel powder and synthetic hydroxyapatite initial dissolution-rate experiments were conducted under various conditions of acid-buffer type and concentration, $\mathrm{pH}$, and common ion concentrations.

In the first paper ${ }^{1}$ of this series, a physicalmodel method for the mechanistic understanding of enamel dissolution was introduced. Using this technic, a detailed analysis of Gray's ${ }^{2}$ extensive data on block-enamel dissolution rates was presented. This first study suggested that one could expect to establish, for example: (1) the mineral phase(s) governing dissolution or chemical reaction; (2) whether the process is solution diffusion controlled or interface reaction rate controlled; (3) the roles played by the acid buffer and $\mathrm{pH}$; (4) the roles of the common ions, calcium and phosphate, and that of fluoride; (5) the nature of possible kinetic barriers arising when adsorbing agents are introduced; (6) whether or not other new surface phases may precipitate during dissolution; and (7) many other facets of the problem depending upon the modifications introduced into the system.

The authors are aware of misconceptions regarding the value of the physical-model method. First, the ultimate objectives were to provide a physically meaningful, unified, quantitative description of the processes through the use of well-established concepts

This investigation was supported by USPHS Research Grant DE-01830 from the National Institute of Dental Research, National Institutes of Health, Bethesda, Md. Received for publication July 8, 1968. of physical chemistry and the accompanying mathematics. The practical value of this is that the simultaneous interactions of many variables can be explained, and predictions can be made in more complex situations, where empirical methods would be of questionable value.

The physical-model method is unique in that every effort is made to give the quantities entering into the mathematical equations well-defined physical meanings, even though in some instances the uncertainties in the actual values may be appreciable. This enables distinction between a failure of a particular assumption in the model and the general uncertainty in the predictions of the model as a whole. Thus logical improvements in the model could be efficiently pursued.

The present report describes the results of continuing studies on the hydroxyapatite model.* A single value for the solubility product, $\mathbf{K}_{\mathrm{sp}}$, of hydroxyapatite used in this model showed good agreement with the buffer concentration, $\mathrm{pH}$, and common ion effects upon the initial dissolution rates in lactic acid buffers. ${ }^{1}$ Some experiments in other acid buffers were also in satisfactory agreement with this model. These findings suggested those experiments that are reported here.

\section{Materials and Methods}

HYDROXYAPATITE MODEL.-As this model has been described in detail earlier, ${ }^{1}$ only an outline will be given here.

Figure 1 presents the basic diffusion controlled dissolution-rate hydroxyapatite model for enamel. When the enamel is exposed to an acid-buffer solution, the acid-buffer mole-

\footnotetext{
* Model $\mathrm{A}$ in reference 1.
} 
cules and hydrogen ions diffuse toward the enamel surface across a liquid diffusion layer of constant thickness, $h$. These acid molecules and ions meet the $\mathrm{PO}_{4}^{3-}, \mathrm{HPO}_{4}^{2-}$, or the $\mathrm{OH}^{-}$that are diffusing outward from the enamel surface. Reactions occur in the liquid diffusion layer to the extent determined by the dissociation constants for the $\mathrm{HPO}_{4}^{2-}$, $\mathrm{H}_{2} \mathrm{PO}_{4}^{-}$, and the buffer acid, as well as by the concentrations of all species involved. If other solution interactions occur, for example, complexation between $\mathrm{Ca}^{2+}$ and the buffer anion, this will also influence the reactions in the diffusion layer. The reaction products then diffuse out of the diffusion layer into the bulk solution along with the $\mathrm{Ca}^{2+}$ and the remaining unreacted phosphate species. For this process, the model assumes that the enamel-solution interface is always in equilibrium with hydroxyapatite, $\mathrm{Ca}_{10}$ $\left(\mathrm{PO}_{4}\right)_{6}(\mathrm{OH})_{2}$. This mineral phase, then, is considered to be responsible for the thermodynamic driving force for dissolution.

The following equations based on simultaneous diffusion and chemical reaction ${ }^{3}$ may translate the foregoing description into mathematical terms:

$$
\begin{gathered}
D_{\mathrm{Cs}} \frac{d^{2}\left[\mathrm{Ca}^{2+}\right]}{d x^{2}}=0 \\
D_{\mathrm{HP}} \frac{d^{2}\left[\mathrm{HPO}_{4}^{2-}\right]}{d x^{2}}-\phi_{1}-\phi_{2}=0 \\
D_{\mathrm{H}_{2} \mathrm{P}} \frac{d^{2}\left[\mathrm{H}_{2} \mathrm{PO}_{-}^{-}\right]}{d x^{2}}+\phi_{1}+\phi_{2}=0 \\
D_{\mathrm{HB}} \frac{d^{2}[\mathrm{HB}]}{d x^{2}}-\phi_{2}+\phi_{3}=0 \\
D_{\mathrm{B}} \frac{d^{2}\left[\mathrm{~B}^{-}\right]}{d x^{2}}+\phi_{2}-\phi_{3}=0 \\
D_{\mathrm{H}} \frac{d^{2}\left[\mathrm{H}^{+}\right]}{d x^{2}}-\phi_{1}-\phi_{3}=0
\end{gathered}
$$

These are expressions for Fick's law ${ }^{4}$ of diffusion in one dimension, taking into account the conservation of mass. Because the diffusion layer thickness is generally much smaller than the dimensions of the surface, it is necessary to consider only one directional coordinate, $x$, which is perpendicular to the surface (Fig 1). The $D$ 's in equations (1-6) are the respective diffusion coefficients for $\mathrm{Ca}^{2+}, \mathrm{HPO}_{4}^{2-}, \mathrm{H}_{2} \mathrm{PO}^{-}$, the buffer-acid molecule $\mathrm{HB}$, the buffer-ion $\mathrm{B}^{-}$, and $\mathrm{H}^{+}$. The bracketed quantities $\left[\mathrm{Ca}^{2+}\right],\left[\mathrm{HPO}_{4}{ }^{2-}\right]$, etc, are the concentrations of the species.

The quantities $\phi_{1}, \phi_{2}$, and $\phi_{3}$ are, respec-

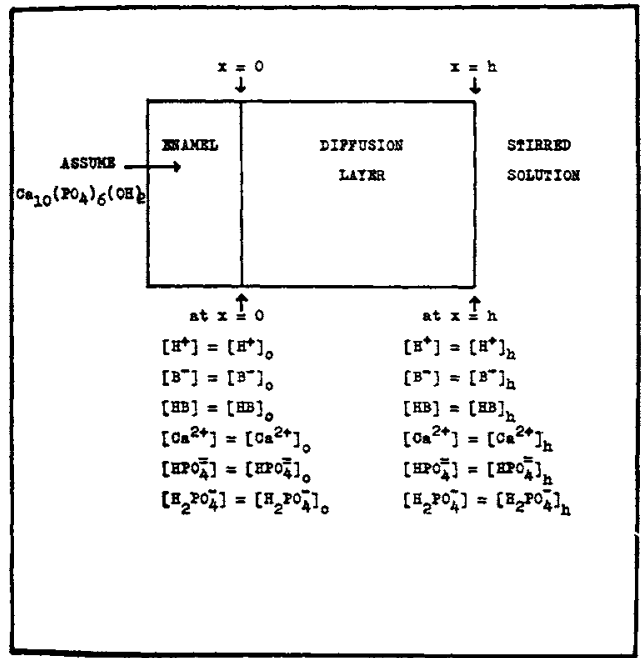

Fig 1.-Hydroxyapatite model for demineralization of dental enamel in acid-buffer solution $\left(\mathrm{H}^{+}\right),\left(\mathrm{Ca}^{2+}\right)$, etc. Solution immediately adjacent to surface is in equilibrium with hydroxyapatite phase.

tively, the rates of reaction per unit volume for the following reactions:

$$
\begin{gathered}
\mathrm{HPO}_{4}{ }^{2-}+\mathrm{H}^{+} \rightarrow \mathrm{H}_{2} \mathrm{PO}_{4}^{-} ; \\
\mathrm{HPO}_{4}{ }^{2-}+\mathrm{HB}^{-} \rightarrow \mathrm{H}_{2} \mathrm{PO}_{4}^{-}+\mathrm{B}^{-} ; \\
\mathrm{H}^{+}+\mathrm{B}^{-} \rightarrow \mathrm{HB} .
\end{gathered}
$$

These reaction rates are assumed to be diffusion controlled. If other reactions of importance occur in the solution phase, equations (1-6) may not describe the situation correctly. Thus, for example, if a calcium complex is present in concentrations comparable to $\mathrm{Ca}^{2+}$, the present equations should be revised to take this factor into account.

In addition to the foregoing equations, the following equilibrium expressions must apply at various places in the diffusion layer:

$$
\begin{aligned}
& \text { at } x>0, K_{\mathrm{B}}=\frac{\left[\mathrm{H}^{+}\right]\left[\mathrm{B}^{-}\right]}{[\mathrm{HB}]} ; \\
& \text { at } x>0, \mathrm{~K}_{2 \mathrm{P}}=\frac{\left[\mathrm{H}^{+}\right]\left[\mathrm{HPO}_{4}^{2-}\right]}{\left[\mathrm{H}_{2} \mathrm{PO}_{4}^{-}\right]} ; \\
& \text {at } x>0, \mathrm{~K}_{3 \mathrm{P}}=\frac{\left[\mathrm{H}^{+}\right]\left[\mathrm{PO}_{4}^{3-}\right]}{\left[\mathrm{HPO}_{4}{ }^{2-}\right]} \\
& \text { at } x=0, \mathrm{~K}_{\mathrm{HAP}}=\left[\mathrm{Ca}^{2+}\right]_{o}^{0}\left[\mathrm{PO}_{4}^{3-}\right]_{o}^{6}\left[\mathrm{OH}^{-}\right]_{o}^{2} \\
& \text { at } x>0, \mathrm{~K}_{w}=\left[\mathrm{H}^{+}\right]\left[\mathrm{OH}^{-}\right] .
\end{aligned}
$$

These $K$ expressions are concentration equilibrium constants determining the relative concentrations of the species at any plane in the diffusion layer. 
Equations (1-6) can now be integrated twice, using the appropriate boundary conditions $^{1}$ and the reaction given by equation (12).

$$
\begin{aligned}
\mathrm{Ca}_{10}\left(\mathrm{PO}_{4}\right)_{6}(\mathrm{OH})_{2}+8 \mathrm{H}^{+} \rightarrow & 10 \mathrm{Ca}^{2+} \\
& +6 \mathrm{HPO}_{4}^{2-}+2 \mathrm{H}_{2} \mathrm{O} .
\end{aligned}
$$

For this purpose it is necessary to assume that the diffusion coefficients and the equilibrium constants are independent of the position in the diffusion layer. This is a fairly good assumption because in most of the present experimental studies, a swamping electrolyte, $\mathrm{NaCl}$, has been used. Even without the added foreign electrolyte, the $D$ 's should be fairly constant at moderate to high buffer concentrations.

The resulting mathematical solution to the above problem is best expressed ${ }^{1}$ by the following two equations:

$$
\begin{aligned}
& \frac{0.6 G h+D_{\mathrm{HP}}\left[\mathrm{HPO}_{4}{ }^{2-}\right]_{h}+D_{\mathrm{H}_{2} \mathrm{P}}\left[\mathrm{H}_{2} \mathrm{PO}_{4}\right]_{h}}{D_{\mathrm{H}_{2} \mathrm{P}}+\left(D_{\mathrm{HP}} K_{2 \mathrm{P}}\right) /\left(\left[\mathrm{H}^{+}\right]_{0}\right)} \\
& =\left[\mathrm{H}_{2} \mathrm{PO}_{4}^{-}\right]_{h}+\frac{D_{\mathrm{H}}}{D_{\mathrm{H}_{2} \mathrm{P}^{2}}}\left[\mathrm{H}^{+}\right]_{h} \\
& -\frac{D_{\mathrm{H}}}{D_{\mathrm{H}, \mathrm{P}}}\left[\mathrm{H}^{+}\right]_{0}+\frac{D_{\mathrm{HiB}}}{D_{\mathrm{H}_{2} \mathrm{P}}}\left[\mathrm{HB}^{-}\right]_{k} \\
& -\frac{D_{\mathrm{HB}}}{D_{\mathrm{H}_{2} \mathrm{P}}}\left\{\frac{D_{\mathrm{HB}}\left[\mathrm{HB}_{{ }_{h}}+D_{\mathrm{B}}\left[\mathrm{B}^{-}\right]_{h}\right.}{D_{\mathrm{HB}}+\left(D_{\mathrm{B}} K_{\mathrm{B}}\right) /\left(\left[\mathrm{H}^{+}\right]_{o}\right)}\right\}-\frac{0.8 G h}{\mathrm{D}_{\mathrm{H}_{2}, \mathrm{P}}}, \\
& {\left[\mathrm{H}^{+}\right]^{1+}=\frac{K_{3}^{\circ} \mathrm{P}}{K_{\mathrm{HAP}}^{2}} K_{\omega}^{2}\left\{\left[\mathrm{Ca}^{2+}\right]_{h}+\frac{G h}{D_{\mathrm{Ca}}}\right\}^{10}} \\
& \times\left\{\frac{0.6 \mathrm{G} h+D_{\mathrm{HP}^{2}}\left(\mathrm{HPO}_{4}^{2--} l_{h}+D_{\mathrm{H}_{2} \mathrm{P}}\left(\mathrm{H}_{2} \mathrm{PO}_{4}^{-}\right]_{h}\right.}{\left(D_{\mathrm{H}_{2} \mathrm{P}} \mathrm{P}\right) /\left(K_{2 \mathrm{P}}\right)+\left(D_{\mathrm{HP}}\right) /\left(\left[\mathrm{H}^{+}\right]_{0}\right)}\right\}^{6} .
\end{aligned}
$$

All the concentration terms with the subscript $h$ are the known bulk-solution concentrations. The only unknowns in equations (13) and (14) are $G, h$, and $\left[\mathrm{H}^{+}\right]_{o} . G$ is the rate of congruent dissolution of the mineral expressed as moles of $\mathrm{Ca}^{2+}$ transported per unit area per unit time; $h$ is the thickness of the diffusion layer; and $\left[\mathrm{H}^{+}\right]_{o}$ is the hydrogen-ion concentration and the enamel surface $(x=0)$. Because $G$ and $h$ always occur together as the product, $G h$, in both equations (13) and (14), one may treat $G h$ as a single unknown. Thus for any selected set of values for the diffusion coefficients, equilibrium constants, and the species concentrations in the bulk solution, one can eliminate $\left[\mathrm{H}^{+}\right]_{o}$ and solve for $G h$ from these two equations (13) and (14).

GENERAL CONSIDERATIONS IN THE DESIGN OF EXPERIMENTS. - The primary objectives of this study were (1) to establish the applicability of the model to data obtained with synthetic apatite preparations and (2) to examine more critically the applicability of the model to experiments with powderedenamel samples.

For the synthetic apatite work, it was decided to conduct experiments with several preparations. Samples developed by researchers at the Tennessee Valley Authority laboratories" were expected to provide the most meaningful tests of the model because these generally always satisfied the highest criteria of crystallinity and purity. It was also decided to conduct dissolution-rate experiments both with powdered hydroxyapatite and with compressed disks of hydroxyapatite. The disk method has the advantage of permitting the direct evaluation of $h$, the diffusion layer thickness. On the other hand, experiments with powdered synthetic apatite were expected to be more convenient for relative rate studies. The powdered-apatite data could also be more conveniently compared with powdered-enamel data.

Enamel dissolution-rate experiments were restricted to powdered enamel in the present study, as powdered enamel generally gives better reproducibility than block enamel when the same batch is used. Also, some block-enamel dissolution-rate data were already available.

Thus these experiments with the hydroxyapatite model were expected to provide a five-way examination of the problem. The powdered-enamel data could simultaneously be analyzed by the model and compared to Gray's' block-enamel data. Independently, the model could be evaluated with the synthetic-apatite data obtained using both powdered apatite and disks of apatite.

ENAMEL POWDER.-The method used for grinding teeth into powder has been described by Hefferren and Higuchi. ${ }^{6}$ Extracted human teeth were cleaned to remove roots, decayed portions, and pulp. These were ground in a model M Fitz mill, * using a 60-mesh screen size. After grinding, the powder obtained was screened, and the fraction collected between 100 to 200-mesh screen size was retained. Then the enamel was separated from the dentin by the flotation procedure described by Manly and Hodge, ${ }^{7}$ using bromoform as the suspending medium. The separated particles were given several rinses with acetone, dried overnight

\footnotetext{
* W. J. Fitzpatrick Co., Chicago, Ill.
} 
in air at room temperature, and finally dried in an oven for one hour at $105 \mathrm{C}$. The chemical analysis showed a calcium to phosphate molar ratio of 1.532 and $73-74 \mathrm{ppm}$ fluoride. ${ }^{\mathrm{s}}$

TVA SAMPLEs.-The procedure ${ }^{5}$ for the preparation of this material involved the reaction of monocalcium phosphate monohydrate and calcium carbonate at high temperatures $(1,200 \mathrm{C})$ in an atmosphere of steam and nitrogen.

The monocalcium phosphate monohydrate and calcium carbonate were ground separately in a glass mortar and sieved through a 200-mesh screen. Stoichiometric quantities, in the ratio of 3 moles of monocalcium to 7 moles of calcium carbonate, were accurately weighed and mixed. The mixture was placed in a covered platinum crucible and inserted in a furnace previously heated to $1,200 \mathrm{C}$. Steam was maintained in the furnace with a Vycor tube, by bubbling nitrogen through water at $96 \mathrm{C}$.

After removing from the furnace and cooling, the material was ground in a glass mortar to pass a 200-mesh screen. This fine powder was extracted in $10-\mathrm{gm}$ portions by shaking with neutral ammonium citrate solution at $55 \mathrm{C}$ for one hour. After decanting, the extraction was repeated to remove all nonapatitic calcium salts. Then the powder was washed several times with water and dried at $120 \mathrm{C}$. The chemical analyses showed a calcium/ phosphate molar ratio of $1.670 \pm 0.01{ }^{*}$ The X-ray diffraction pattern compared very well with that for the sample obtained from the TVA laboratories.

VICTOR SAMPLES.-A sample of Tricalcium Phosphate $\dagger$ was also used to a limited extent. The powder was first slugged, using a Carver laboratory press $\$$ at $75,000 \mathrm{psi}$ pressure and then ground lightly in a glass mortar. After grinding, the granules were screened, and the fraction collected between size 80 - and 100-mesh screens was retained. The sample had a calcium/ phosphate molar ratio of 1.625 . $^{\circ}$

Preparation of BUfFers.-Acetate and lactate buffers were prepared by mixing calculated quantities of the acids and their sodium salts. Because concentrated lactic acid contains polymers of lactic acid, diluted

\footnotetext{
* Based on calcium analysis by gravimetric method $=100.5 \pm 0.5 \%$ and phosphate analysis ${ }^{8}=100.0 \pm 1.0 \%$

$\uparrow$ Stauffer Chemical Co., Victor Division, Chicago, Ill.

t. Fred S. Carver Laboratories Inc. Summit, NJ.
}

lactic acid solutions were allowed to age 2 for one month. Calculated amounts of sodium chloride were added whenever it was necessary to obtain a desired ionic strength of the final solution. After mixing the free acid, its sodium salt, and the sodium chloride, the $\mathrm{pH}$ was measured in all instances, using a Beckman Model $\mathrm{G}$ pH meter at room temperature.

Buffers used in the common ion studies were prepared by adding predetermined amounts of calcium chloride or potassium dihydrogen phosphate.

ANALYTIC PROCEDURES.-Phosphate concentrations were determined in all experiments by the method of Gee, Domingues, and Deitz, ${ }^{9}$ in which the phosphoammonium molybdate complex formed was reduced by stannous chloride. The absorbance of the resulting blue color was determined at the end of 15 minutes at $\lambda=720 \mathrm{~m} \mu$.

Calcium concentrations were determined either by a colorimetric method ${ }^{10}$ or by atomic absorption. $\S$ The colorimetric method involved measuring the absorbance of a complex formed between calcium ions and the reagent, calcichrome, $\|$ at $\lambda=510 \mathrm{~m} \mu$ and $\mathrm{pH}=12.0$. Because phosphate interfered to some extent, the procedure was modified by adjusting the phosphate concentrations to a constant level in both the standards and the unknown solutions.

In the atomic-absorption procedure for calcium, known aliquots of standard calcium solutions\# and unknown sample solutions were pipetted into $25-\mathrm{ml}$ volumetric flasks. Because of phosphate interference at the higher phosphate concentrations ( 70 to 100 ppm), $2.5 \mathrm{ml}$ of a $10 \%$ strontium chloride solution was added to each flask. It was also found necessary to add the same quantity and molarity of the acetate buffer to the standard calcium solutions as was present in the experimental runs. The volumes were made up to $25 \mathrm{ml}$ with distilled water. The solutions were then aspirated into the acetylene-air-mixture flame of the atomic-absorption spectrophotometer Model 303 and the absorbances at $422.7 \mathrm{~m} \mu$ were determined.

EXPERIMENTAL PROCEDURE FOR DISSOLUTION-RATE STUDIES OF ENAMEL POWDER OR SYNTHETIC HYDROXYAPATITE POWDER.-A

\footnotetext{
$\S$ Perkin EImer Corporation, Norwalk, Conn.

\| Burdick and Jackson Laboratories, Inc, Muskegon, Mich.

\#Fischer Scientific Co., Fairlawn, NJ.
} 
typical run involved the following: A 100$\mathrm{mg}$ sample of the enamel powder or the synthetic hydroxyapatite powder was transferred to a $250-\mathrm{ml}$ volumetric flask clamped to the arm of a Burrell wrist action shaker. This flask was more than $50 \%$ immersed in a water bath maintained at $30 \mathrm{C} \pm 0.2 \mathrm{C}$. Then $200 \mathrm{ml}$ of the required buffer, already equilibrated at $30 \mathrm{C}$, was transferred, and at the same time the shaker and a timer was started. The constant speed of the shaker was set high enough so that the powder particles were maintained in a suspended state in the solution. At various intervals, 5- or $10-\mathrm{ml}$ samples were withdrawn by means of a hypodermic syringe* and needle and were filtered through Millipore $\dagger$ filters $(0.22-\mu$ pore size) in Swinny Filter holders. These samples were then analyzed for both calcium and phosphate. In some cases, $75 \mathrm{mg}$ of the sample was used, in which cases the buffer used was $150 \mathrm{ml}$.

In the TVA powder experiments, most of the studies were conducted using samples that were about $15 \%$ predissolved in an acetate buffer. The reason for using predissolved samples was that it was rather difficult to obtain initial slopes because of an early abrupt curvature in the amount dissolved of hydroxyapatite versus time plots.

Another technic used for withdrawing samples involved a syringe-filter assembly consisting of a glass filter stick (maximum pore size of 4 to $8 \mu$ ) connected by Tygon tubing to the inlet of a B.D. $\ddagger$ automatic valve attached to a $50-\mathrm{ml}$ syringe. The outlet of the valve was connected to a piece of latex-rubber tubing which dipped back into the solution. This arrangement permitted sampling and then rinsing out the line before the next sample was taken.

EXPERIMENTAL PROCEDURE FOR DISSOLUTION-RATE STUDIES WITH SYNTHETIC-APATITE DISKS.-An apparatus ${ }^{11}$ which affords rigid geometry and reproducible hydrodynamics was employed (Fig 2). Six flat round disks $(1 / 2$ " diameter) of hydroxyapatite were mounted by means of wax in the holes of lucite baffles mounted at the bottom of a beaker (diameter, $3^{\prime \prime}$; height, $8^{\prime \prime}$ ). Thus only one side of these disks was exposed to the solution medium. A three-bladed polyethy-

\footnotetext{
* Becton, Dickinson \& Co., Rutherford, NJ.

$\dagger$ Millipore Filter Corporation, Bedford, Mass.

\$ Becton, Dickinson \& Co., Rutherford, NJ.
}

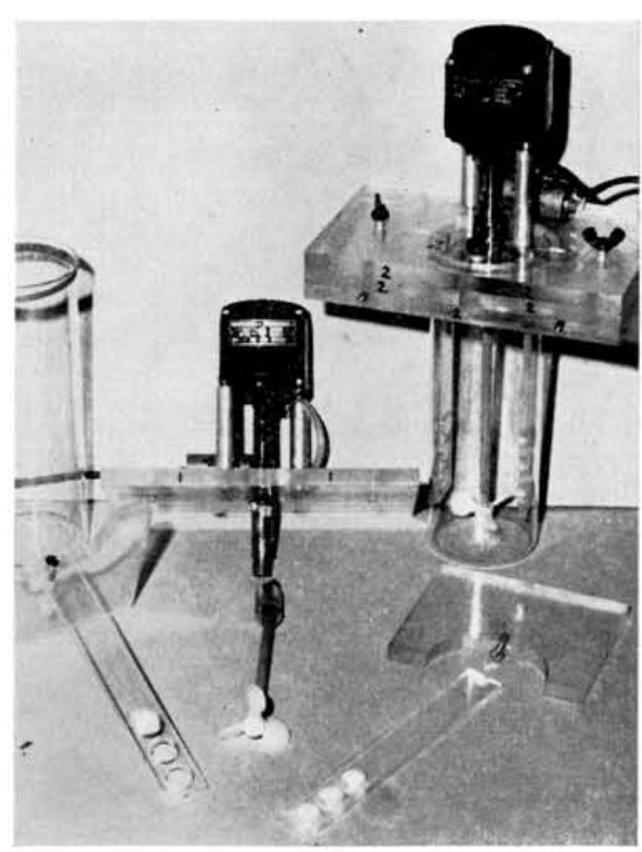

FIG 2.-Photograph of assembled and disassembled dissolution apparatus. Assembled unit is suspended above a water bath with beaker projecting down into the bath.

lene propeller directly coupled to a 300-rpm synchronous motor provided constant agitation.

Buffer $(425 \mathrm{ml})$ was pipetted into the dissolution chamber and allowed to come to thermal equilibrium at $30 \mathrm{C}$. The solution was stirred during this period. When the buffer had reached $30 \mathrm{C}$, the motor-stirrer assembly was lifted, and the baffle assemblies, containing six disks, were inserted into the solvent. The apparatus was immediately clamped together. Simultaneous to the initiation of stirring, a stopwatch was started.

A $25-\mathrm{ml}$ sample was withdrawn by pipet as soon after the initiation of stirring as practical (usually three minutes) for use as a control. Periodically thereafter, samples were withdrawn by a volumetric pipet and diluted for analysis. The sample volume was not replaced.

PROCEDURE FOR COMPARING THE THEORY WITH THE EXPERIMENTAL INITIAL DISSOLUTION RATES FOR POWDERED SAMPLES.-For all the rate experiments involving powdered enamel, the initial dissolution rates were determined from the amount of hydroxy- 
apatite dissolved versus time plots in the following manner. Whenever possible, the slopes $(\mathrm{gm} / \mathrm{sec})$ were graphically taken at the point where $10 \%$ of the mineral had dissolved. These were then corrected for the reduction in driving force for dissolution estimated from the amount dissolved at that time and the amount at equilibrium, that is, at long intervals. When there were not enough data points to cover the region up to $10 \%$ dissolution, slopes were taken at $5 \%$ dissolved and corrected as before. In some cases, the initial rates were estimated from extrapolation of the data points to small time values.

Another procedure for calculating the initial dissolution rates was used for the TVA powder samples. For the different concentrations of acetate buffer at a single $\mathrm{pH}$, the slopes of the amount dissolved versus time plots were compared at the same percentage dissolved of hydroxyapatite. For example, consider the dissolution data of TVA sample in $\mathrm{pH} 4.5$ acetate buffer (Fig 9) and calculate the relative rates of $0.01 \mathrm{M}$ and $0.05 \mathrm{M}$ acetate buffers. One can plot all initial points up to 1,800 seconds of dissolution for this set and draw smooth curves through them. Then one may compare the slopes at $2 \%$ dissolved, at $3 \%, 4 \%$, and so on.

The two procedures used to calculate initial rates agreed rather well when comparisons between the two technics were made.

In order to compare the theoretically computed $G h$ values with the experimental dissolution rate, $G e$, the following relationships must be considered:

$$
G h=\frac{[G e][h]}{[A]},
$$

where $A=$ surface area of $100 \mathrm{mg}$ of the powdered enamel used in the experiment. Therefore the experimental rate is related to the theoretical $G h$ by a factor, $k=\frac{A}{h}$, which should be a constant for initial rates.

PROCEDURE FOR COMPARING THE THEORY WITH THE EXPERIMENTAL INITIAL RATES USING THE DISK METHOD.-The initial rates, $G e$, were obtained from the initial slopes of the amount dissolved versus time plots, and then equation (15) was used for comparing the experimental rate with the theoretical $G h$ values. The particular advantage of the disk method is that the effective aqueous diffusion layer, $h$ (equations [13] and [14]), may be determined independently for a given apparatus. This was done ${ }^{11}$ for the present system, using benzoic acid disks for which the surface area, solubility, and the diffusion coefficient were known. The theoretical Gh values were computed, using equations (13) and (14) and the same parameters given in Table 1.

\section{Results}

EXPERIMENTAL RESULTS WITH POWDERED ENAMEL.-Typical data obtained with powdered enamel in the dissolution-rate experiments are shown in Figures 3 and 4. These are based on phosphate analyses. Although the precision was somewhat less, essentially the same results were obtained with calcium analyses. Except in the dissolution experiments in the presence of a common ion, both the calcium and the phosphate analyses were always carried out, and in all cases congruent dissolution was generally observed. Only representative raw data are presented here.*

From the raw dissolution data (Fig 3 and 4 ), initial dissolution rates were determined. These were expected to be the most meaningful quantities for comparison between data and model. For initial rates, the changes in surface area for a given batch of enamel sample or the changes in the solution concentration of species with time were not a factor. Also, for initial rates the effective hydrodynamic factor, $h$, was a constant because the initial particle-size distribution was the same for all runs. Thus initial rates were expected to be directly comparable to the predictions of the model regarding buffer

* Additional information is available on request to the authors.

TABLE 1

EQUILIBRIUM CONSTANTS FOR Theoretical Calculations

\begin{tabular}{lll}
\hline \multicolumn{1}{c}{ Parameter } & \multicolumn{1}{c}{$\begin{array}{c}\text { Thermodynamic } \\
\text { Constants }\end{array}$} & $\begin{array}{c}\text { Corrected } \\
\text { Constants }\end{array}$ \\
\hline$K_{2 \mathrm{P}}$ & $6.3 \times 10^{-8}$ moles $/ 1$ & $2.0 \times 10^{-7}$ \\
$K_{3 \mathrm{P}}$ & $4.7 \times 10^{-13}$ & $1.4 \times 10^{-12}$ \\
$K_{w}$ & $2.6 \times 10^{-14}+$ & $4.1 \times 10^{-14}$ \\
$K_{\mathrm{B}}$ (acetic) & $1.75 \times 10^{-5}$ & $2.2 \times 10^{-5}$ \\
$K_{\mathrm{B}}$ (lactic) & $1.39 \times 10^{-4}$ & $2.0 \times 10^{-4}$ \\
$K_{\mathrm{HAP}}$ & $1 \times 10^{-120}$ & $\ldots \ldots \ldots$ \\
$K^{6}{ }_{3 \mathrm{P}} K^{2}{ }_{w} / K_{\text {HAP }}$ & $1 \times 10^{-124}$ & $1.6 \times 10^{11}+$ \\
& $\ldots \ldots \ldots \ldots$ & $1.6 \times 10^{15}$ \\
\hline
\end{tabular}

* Based upon activity coefficient correction estimated for ionic strength $=0.1-0.2$.

+ Based on $37 \mathrm{C}$

$¥$ Based on $K_{\text {HAP }}=1 \times 10^{-120}$.

Based on $K_{\mathrm{HAP}}^{\mathrm{HAP}}=1 \times 10^{-124}$. 


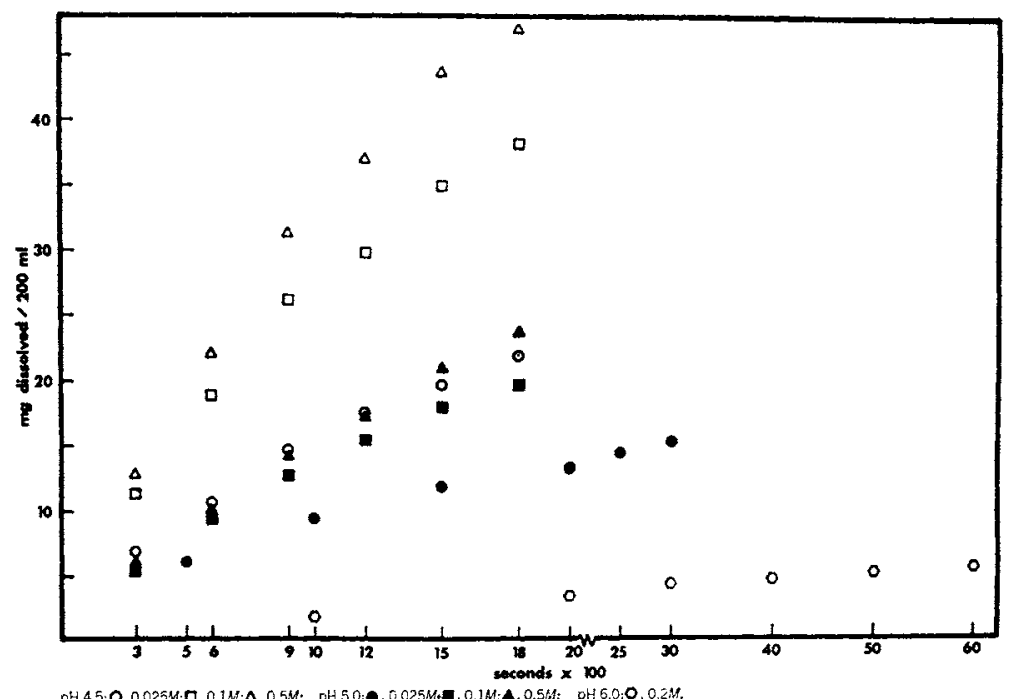

FIG 3.-Dissolution of enamel powder in $\mathrm{pH} 4.5,5.0$, and 6.0 with different concentrations of acetate buffers without sodium chloride.

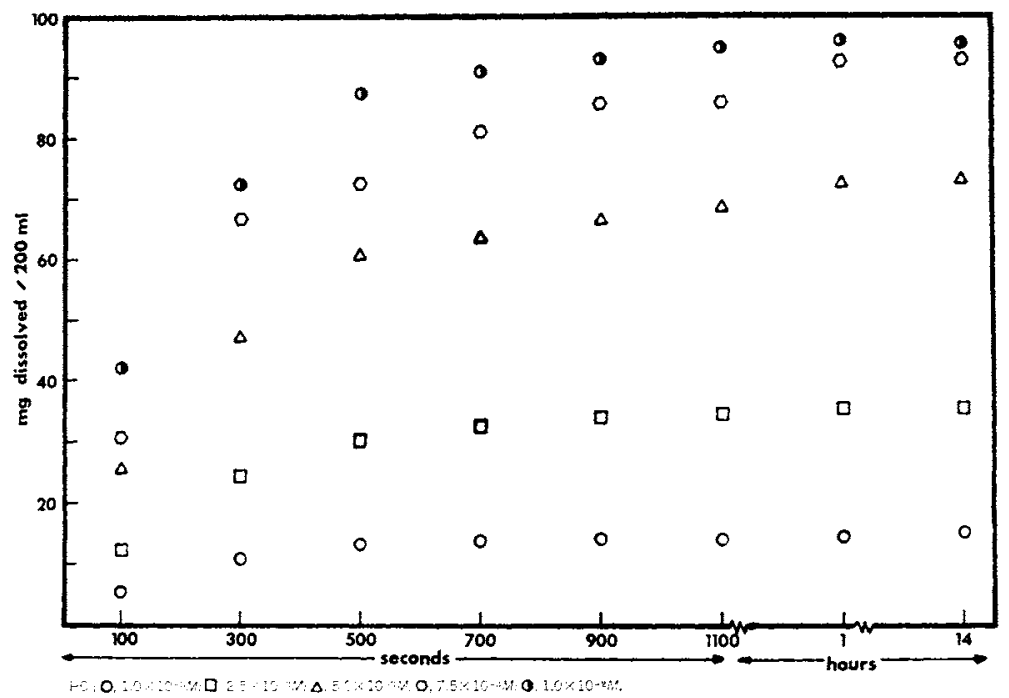

FIG 4.-Dissolution of enamel powder in hydrochloric acid with $0.5 M$ $\mathrm{NaCl}$.

type, buffer concentration, $\mathrm{pH}$, and common ion concentration dependencies.

COMPARISON OF POWDERED-ENAMEL DATA WITH MODEL.- The experimental initial rates are presented in Figures 5 and 6 . These figures show the buffer concentration and $\mathrm{pH}$ dependencies in acetate and lactate buffers with and without added $0.5 \mathrm{M}$ sodium chloride. Figures 7 and 8 present the common ion effects in the two buffers. Here the results are expressed as percent of the zero common ion rate.

Table 1 gives the values for the various parameters of theoretical calculations of $G h$ used with equations (13) and (14). The corrected constants in Table 1 were obtained by "correcting" the thermodynamic constants with selected activity coefficients. ${ }^{1}$ Admitted- 


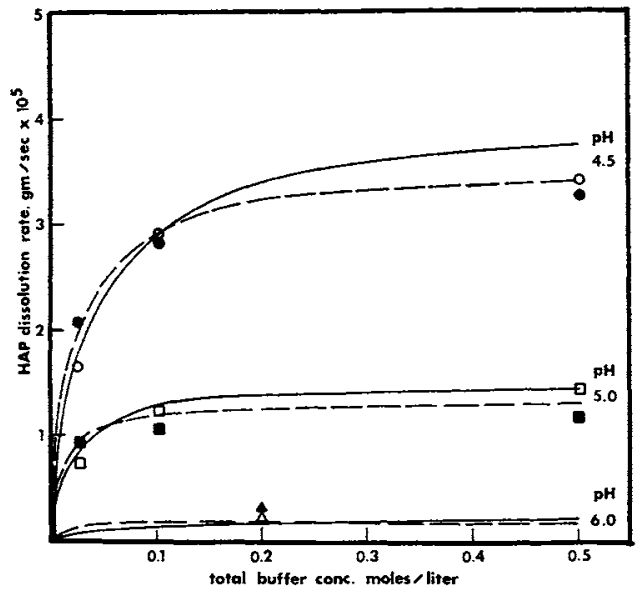

Fig 5.- Initial dissolution rates of enamel powder in acetic acid buffers. Smooth curves correspond to theory with $K_{\mathrm{EAP}}=1.0 \times 10^{-120}$, and $k=4.4 \times 10^{3}$. Broken curves correspond to theory with $K_{\mathrm{HAP}}=1.2 \times 10^{-124}$ and $k=7.0 \times 10^{3}$. The three sets of points are the experimental values at the three different $\mathrm{pH}$ conditions. Closed symbols correspond to the presence of $\mathrm{NaCl}$ in the buffers, and open symbols correspond to the absence of $\mathrm{NaCl}$.

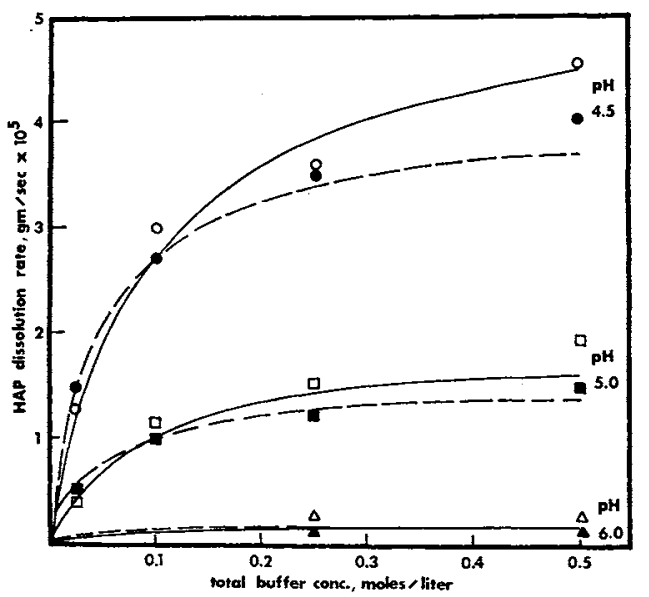

FIG 6.--Initial dissolution rates of enamel powder in lactic acid buffers. Smooth curves correspond to theory with $K_{\mathrm{HAP}}=1.0 \times 10^{-120}$ and $k=5.7 \times 10^{3}$. Broken curves correspond to theory with $K_{\mathrm{HAP}}=1.0 \times 10^{-124}$ and $k=8.0 \times 10^{3}$. The three sets of points are the experimental values at different $\mathrm{pH}$ conditions. Closed symbols correspond to the presence of $\mathrm{NaCl}$ in the buffers, and open symbols correspond to the absence of $\mathrm{NaCl}$.

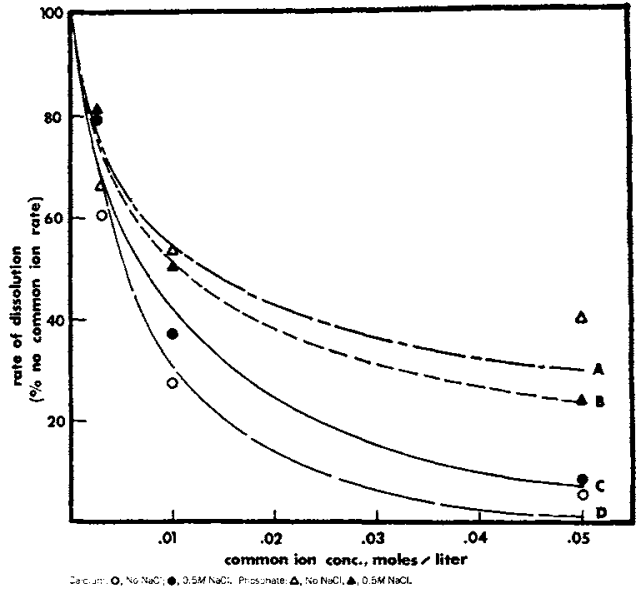

Fig 7.-Effect of the common ions, calcium and phosphate, on the initial dissolution rates of enamel powder at $\mathrm{pH} 4.5$ and $0.05 \mathrm{M}$ total acetate-buffer concentration. Curves $A$ and $B$ correspond to theory for phosphate common ion when $K_{\text {HAP }}=1 \times 10^{-120}$ and $1 \times 10^{-124}$, respectively. Curves $C$ and $D$ correspond to theory for calcium common ion when $K_{\mathrm{HAP}}=1 \times 10^{-120}$ and $1 \times 10^{-124}$, respectively.

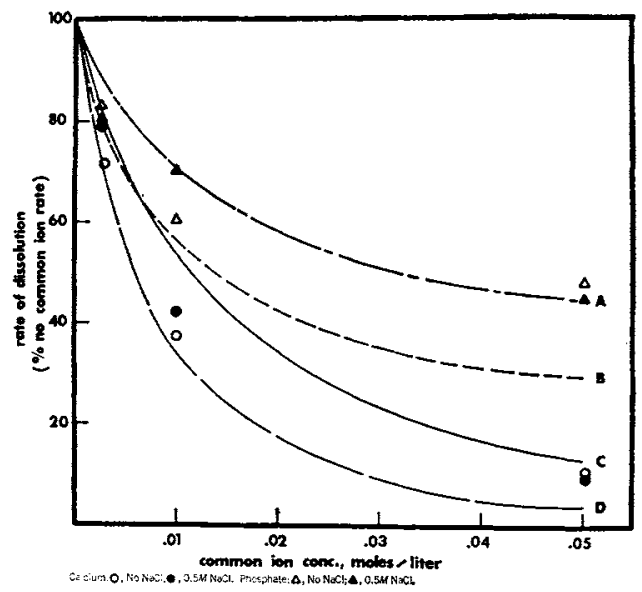

FIG 8.-Effect of calcium and phosphate on the initial dissolution rates of enamel powder at $\mathrm{pH} 4.5$ and $0.05 \mathrm{M}$ total lactate-buffer concentration. Curves $A$ and $B$ correspond to theory for phosphate common ion when $K_{\mathrm{HAP}}=1.0 \times 10^{-120}$ and $1.0 \times 10^{-124}$, respectively. Curves $C$ and $D$ correspond to theory for calcium common ion when $K_{\text {HAP }}=1.0 \times 10^{-120}$ and $1.0 \times 10^{-124}$, respectively. 
$1 y$, for the high ionic strengths encountered in these studies, such corrections are open to certain questions. ${ }^{1}$ Except for $D_{\mathrm{H}+}$, the diffusion coefficients entering into equations (13) and (14) were all assumed to be equal to $1 \times 10^{-5} \mathrm{~cm}^{2} / \mathrm{sec}$. In the acetate and lactate buffered cases the transport of hydrogen ions was not important, whereas in the dissolution experiments involving hydrochloric acid, the choice of the value of $D_{\mathrm{H}^{+}}$was expected to be important.

The curves in Figures 5 and 6 are the theoretically computed dissolution rates. The smooth curves are for $K_{\mathrm{HAP}}=1 \times 10^{-120}$, and $k=5.7 \times 10^{3}$ and $k=4.4 \times 10^{3}$ for the lactate and the acetate buffers, respectively. The broken curves correspond to $K_{\mathrm{HAP}}=1 \times 10^{-124}$, and $k=8.0 \times 10^{3}$ and $k=7.0 \times 10^{3}$ for the lactate and the acetate buffers, respectively.

The theoretical predictions of the common ion effects were calculated, using the same equations (13) and (14) and the parameters given in Table 1. However, in this case, it was not necessary to involve $k$ because this quantity cancels in the type of relative plots given in Figures 7 and 8 . The smooth curves are for $K_{\mathrm{HAP}}=1 \times 10^{-120}$, and the broken curves are for $K_{\mathrm{HAP}}=1 \times 10^{-124}$.

It can be seen from Figures 5-8 that the model predictions are generally in good agreement with the experimental $\mathrm{pH}$, the buffer concentration, and the common ion dependencies in both acetate and lactate buffers. Two items should be noted in this regard. First, the model was not very sensitive to changes in the order of $10^{4}$ in $K_{\text {H.AP }}$. Thus both $K_{\mathrm{HAP}}=1 \times 10^{-320}$ and $K_{\mathrm{HAP}}=$ $10^{-124}$ gave apparently good fits to the data when the appropriate $k$ values were employed. It appeared that the best $K_{\text {HAP }}$ value lay somewhere in this neighborhood. Second, the best $k$ values for acetate and lactate buffers appeared to be somewhat different when using either $K_{\mathrm{HAP}}=1 \times 10^{-120}$ or $K_{\mathrm{HAP}}$ $=1 \times 10^{-124}$. These differences were almost of the same order of magnitude as the experimental uncertainty. Table 2 gives the

TABLE 2

$k$ VALUES FOR ENAMEL

\begin{tabular}{lcc}
\hline \hline Buffer Type & $K_{\mathrm{HAP}}=10^{-120}$ & $K_{\mathrm{HAP}}=10^{-124}$ \\
\hline Acetate & $4.4 \times 10^{3}$ & $7.0 \times 10^{3}$ \\
Lactate & $5.7 \times 10^{3}$ & $8.0 \times 10^{3}$ \\
\hline
\end{tabular}

$k$ values which are dependent on the choice of $K_{\mathrm{HAP}}$. For $K_{\mathrm{HAP}}=10^{-124}$, the average $k$ value was $7.5 \times 10 .^{3}$

EXPERIMENTAL RESULTS WITH TVA POWDER.--The experimental data of $15 \%$ predissolved TVA powder sample showing dependency on $\mathrm{pH}$ and acid-buffer concentration on its dissolution are presented in Figure 9. In these buffers, the total ionic strength was maintained at $0.5 \mathrm{M}$ with the addition of sodium chloride. These particular data were based on phosphate analyses. However, calcium analyses were always done, and the dissolution was always found to be congruent.

The experimental initial dissolution rates. shown in Figure 10, were determined from the amount of hydroxyapatite dissolved versus time plots (Fig 9), particularly from the first 800 to 1,800 seconds data points, where only 5 to $10 \%$ of the solid had dissolved. For these time periods, the concentration buildup of the reaction products in the bulk solution was small enough so that this may be neglected.

COMPARISON OF TVA POWDER SAMPLE DATA WITH MODEL.- The theoretical calculations of $G h$ were made by means of equations (13) and (14), as was done in the powdered-enamel case. The curves in Figure 10 give the theoretically computed rates. The smooth curves are for $K_{\mathrm{HAP}}=$ $10^{-131}$ and $k=2.0 \times 10^{4}$ and the broken ones for $K_{\mathrm{HAP}}=10^{-132}$ and $k=2.3 \times 10^{4}$. Figure 11 shows the comparison for the experimental common ion effects with the theoretical model predictions. The smooth curves here are for $K_{\mathrm{HAP}}=1 \times 10^{-132}$, and the broken curves correspond to $K_{\mathrm{HAP}}=1 \times$ $10^{-131}$. As mentioned earlier, for the case of common ion effects, the theoretical predictions do not involve $k$ because this quantity cancels in this type of plot (Fig 11).

Figures 10 and 11 show that the model predictions agree very well with the experimental data for $\mathrm{pH}$, acid-buffer concentration, and common ion dependencies on the TVA powder dissolution rates when a $K_{\text {HAP }}$ value of $1 \times 10^{-131}$ to $1 \times 10^{-132}$ is employed.

EXPERIMENTAL RESULTS WITH TVA USING THE DISK METHOD AND ITS COMPARISON WITH MODEL.-Figure 12 shows the experimental data for the dissolution of TVA disks in $\mathrm{pH} 5.0$ and 5.5 in varying concentrations 


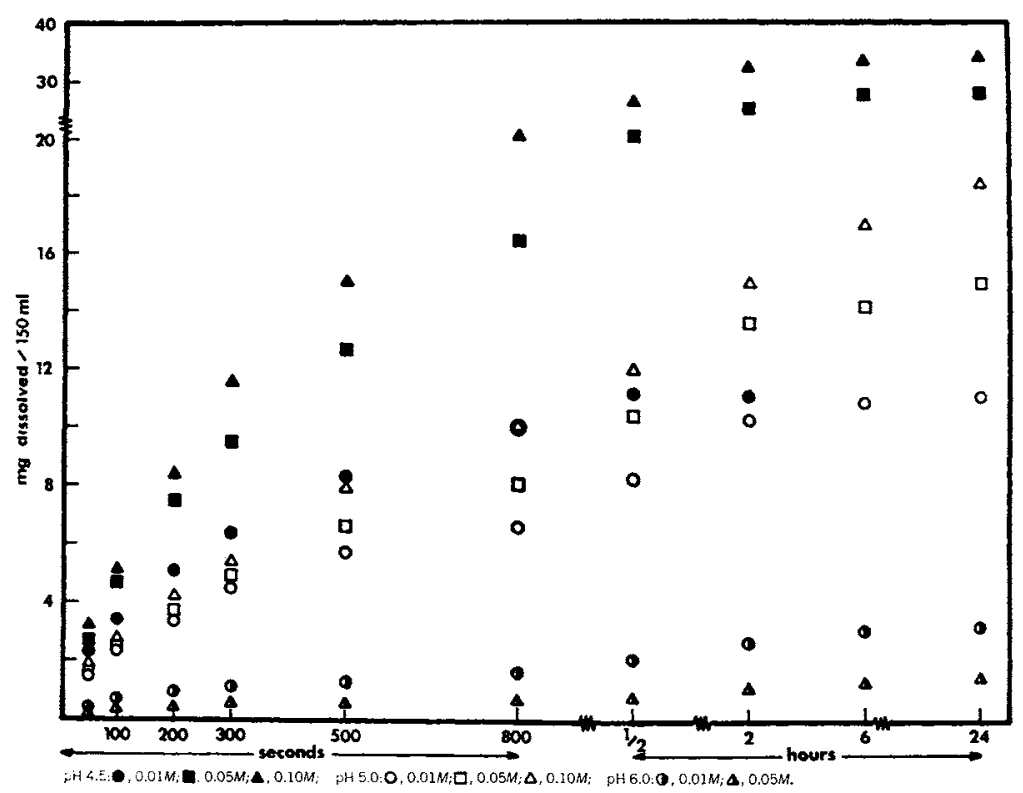

FIG 9.-Dissolution of $15 \%$ predissolved TVA apatite powder in $\mathrm{pH} 4.5$, 5.0 , and 6.0 at different concentrations of acetate buffers. The total ionic strength of the buffers was maintained at $0.5 \mathrm{M}$ with $\mathrm{NaCl}$.

of acetate buffers. The total ionic strength in all the buffers was adjusted to $0.5 M$ with the addition of sodium chloride. The initial dissolution rate, $G$, was then calculated from the data in Figure 12 by determining the initial slopes from the plots.

Figures 13 and 14 show comparisons of the experimental and theoretical $G h$ values. The experimental $G h$ was obtained by multiplying the experimental $G$ determined above with $h=25.5 \times 10^{-4}$ which was found ${ }^{11}$ for the conditions in these studies. The theoretical results for two $K_{\mathrm{HAP}}$ values are given in these figures.

As can be seen, the agreement between the theoretical model predictions using a $K_{\mathrm{HAP}}$ value of around $1 \times 10^{-129}$ to $1 \times 10^{-131}$ are in very good agreement with the experiments. The TVA powder experiments agreed very well with the same model ( $K_{\mathrm{HAP}}$ $=1 \times 10^{-130}-1 \times 10^{-132}$ ).

EXPERIMENTAL RESULTS WITH THE VICTOR POWDER SAMPLE AND ITS COMPARISON WITH MODEL.-Figure 15 shows the dependency of the initial dissolution rates of Victor sample upon the $\mathrm{pH}$ and the acetate-buffer concentration. The solid theoretical curves in this figure are when $K_{\mathrm{HAP}}=1 \times 10^{-116}$ and $k=1.34 \times 10^{4}$ and the broken curves for $K_{\mathrm{HAP}}=1 \times 10^{-108}$ and $k=5.8 \times 10^{3}$. The open experimental points are for no sodium chloride in the dissolution medium, and the closed points refer to $0.5 \mathrm{M}$ sodium chloride. It appeared from this figure that Victor sample agreed rather well with $K_{\mathrm{HAP}}=1 \times$ $10^{-116}$ and $k=1.34 \times 10^{4}$ in all the $\mathrm{pH}$ instances.

It is noteworthy that in the common ion experiments in both lactate and acetate buffers, the Victor samples were also found ${ }^{8}$ to be in approximate agreement with the model. However, greater discrepancies from the theoretical predictions were observed than with either the enamel powder or the TVA sample experiments.

\section{Discussion}

Powdered ENAMEl STUdies IN ACETATE AND LACTATE BUFFER SYSTEMS.-The fact that all the powdered-enamel experimental data were found to be in good agreement with the hydroxyapatite model is very significant for a number of reasons. The predictions of the model with $K_{\mathrm{HAP}} \cong 1 \times 10^{-120}$ $-1 \times 10^{-124}$ were consistent with data (Fig $5,6)$ obtained with two buffer systems, acetate and lactate, at several buffer con- 


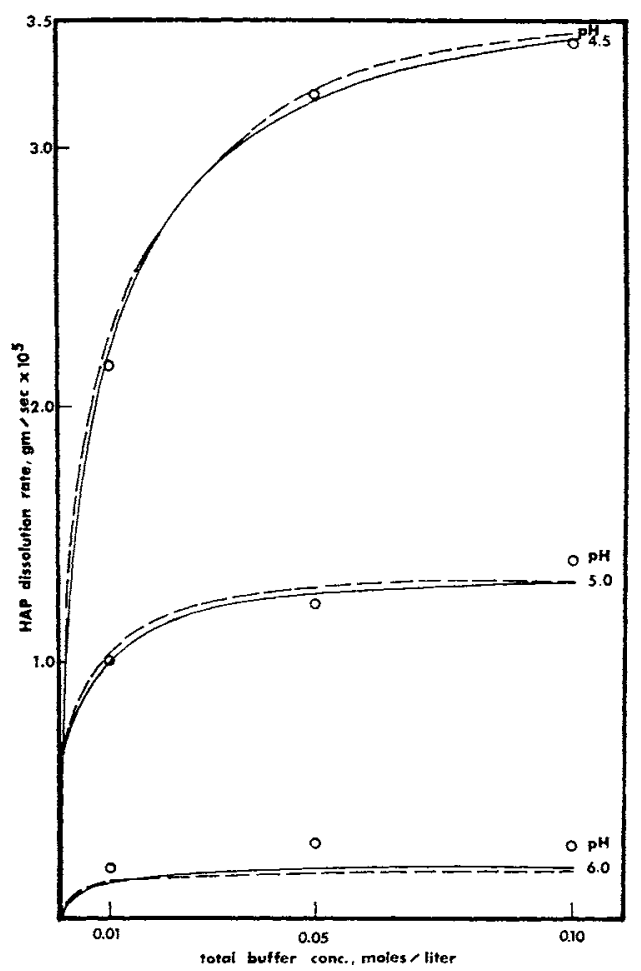

FIG 10.--Initial dissolution rates of $15 \%$ predissolved TVA apatite powder in acetic acid buffers. Smooth curves correspond to theory with $K_{\mathrm{HAP}}=1.0 \times 10^{-131}$ and $k=2.0 \times 10^{4}$. Broken curves correspond to theory with $K_{\mathrm{HAP}}=1.0 \times$ $10^{-132}$ and $k=2.3 \times 10^{4}$. The three sets of points are the experimental values at different $\mathrm{pH}$ conditions.

centrations and $\mathrm{pH}$ values. Furthermore, both the calcium common ion and the phosphate common ion experimental results (Fig $7,8)$ also agreed with the theoretical predictions. This serves as an independent test of the model.

It is particularly important to note that this same model with the same $K_{\mathrm{HAP}}$ values were found to describe best the extensive experimental data on block-enamel dissolution rates in weak acid buffers obtained by Gray. ${ }^{2}$ Gray had experimentally studied the $\mathrm{pH}$, the buffer concentration, the buffer type, and the common ion effects upon block-enamel dissolution. Essentially all his data conformed to the predictions of the present theory.

POWDERED-ENAMEL DISSOLUTION BEHAVIOR IN $\mathrm{HCl}$. - The data on the powderedenamel dissolution in hydrochloric acid solu- tions (Fig 4) was not discussed because at good choice for the effective diffusion coefficient of the hydrogen ion was not apparent. The value of $1 \times 10^{-5} \mathrm{~cm} \% / \mathrm{sec}$ is not a reasonable one ${ }^{12}$ for $\mathrm{H}^{+}$.

Figure 16 presents a comparison of the experimental rates for powdered enamel in hydrochloric acid solutions obtained from the data in Figure 4 with the hydroxyapatite model predictions. The theoretical curve may be calculated from the general equations (13) and (14), using $K_{\mathrm{HAP}}=1 \times 10^{-121}$ with $k D_{m+}=0.65$ or $K_{\mathrm{HAP}}=1 \times 10^{-12}$ with $k D_{\mathrm{H}^{+}}=0.66$.

First, it should be noted that the $\mathrm{HCl}$ concentration dependence of the experimental data is in good agreement with theory. Second, a value for $D_{\mathrm{H}-}$ may be estimated because $k$ may be obtained from the acetate and lactate systems. For $K_{\mathrm{HAP}}=$ $1 \times 10^{-124}$, the average $k$ value is $7.5 \times 10^{3}$. This then gives $D_{\mathrm{H}^{+}}=8.8 \times 10^{-\pi} \mathrm{cm}^{2} / \mathrm{sec}$. For $K_{\mathrm{H} .1 \mathrm{P}}=1 \times 10^{-120}, k=5.0 \times 10^{3}$ and $D_{\mathrm{H}+}$ $=1.3 \times 10^{-4} \mathrm{~cm}^{2} / \mathrm{sec}$. These $D_{\mathrm{H}^{+}}$values are five to ten times greater than diffusion coefficients expected for most small molecules and ions and agree well with the studies by Vinograd and $\mathrm{McBain}^{12}$ on the diffusion behavior of $\mathrm{H}^{+}$in $\mathrm{HCl}-\mathrm{NaCl}$ mixtures. Thus the experiments on the dissolution-rate behavior in strong acid solutions have also been found to be consistent with the theory.

TVA APATITE STUDIES.-The hydroxyapatite model has been able to explain consistently all the experimental data for the TVA sample used in these studies. Both the powder method and the disk method yielded data on the buffer concentration. $\mathrm{pH}$, and common ion dependencies that are in quantitative agreement with the model using $K_{\mathrm{HAP}}$ value of around $1 \times 10^{-131}$. It is noteworthy that the two experimental methods and the common ion studies represent three independent tests of the model. Thus the overall good consistency is quite significant and strongly supports the general applicability of the theoretical model with $K_{1, \mathrm{PP}} \cong 1 \times 10^{-130}-1 \times 10^{-132}$.

\section{Conclusions}

This work was an attempt to study critically the hydroxyapatite model for the dissolution rate of enamel in acidic media which was recently proposed. ${ }^{1}$ Experiments were conducted with various samples of 


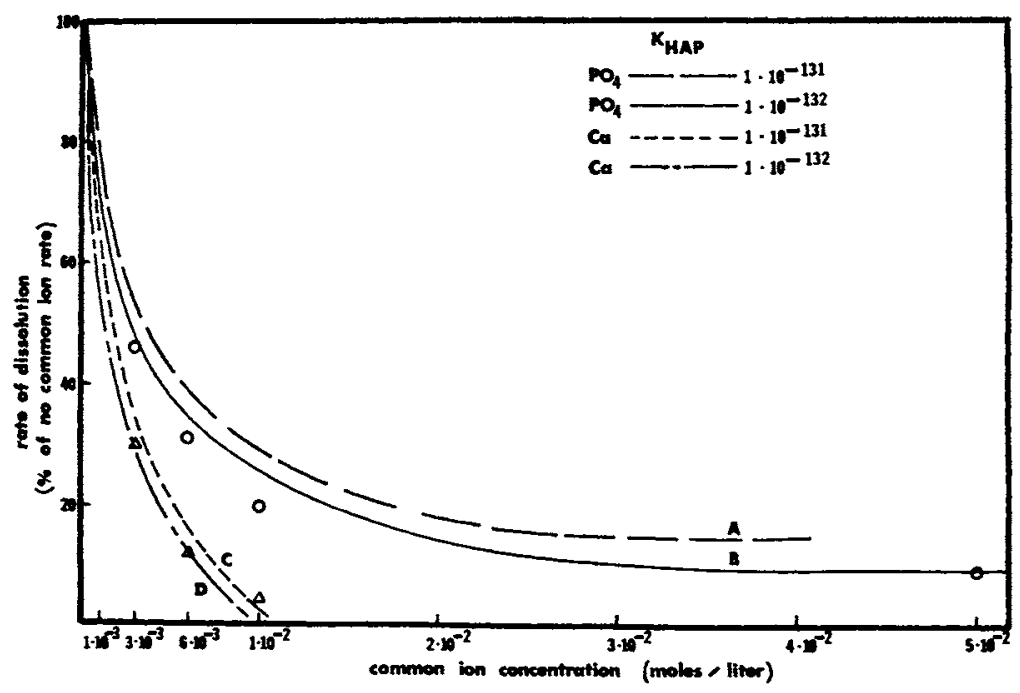

FIG 11.-Effect of calcium and phosphate on the initial dissolution rates of $15 \%$ predissolved TVA apatite powder at $\mathrm{pH} 4.5$ and $0.05 \mathrm{M}$ total acetatebuffer concentration. Curves $A$ and $B$ correspond to theory for phosphate common ion when $K_{\mathrm{HAP}}=1 \times 10^{-131}$ and $1 \times 10^{-132}$, respectively. Curves $C$ and $D$ correspond to theory for calcium common ion when $K_{\mathrm{HAP}}=1$ $\times 10^{-131}$ and $1 \times 10^{-132}$, respectively.

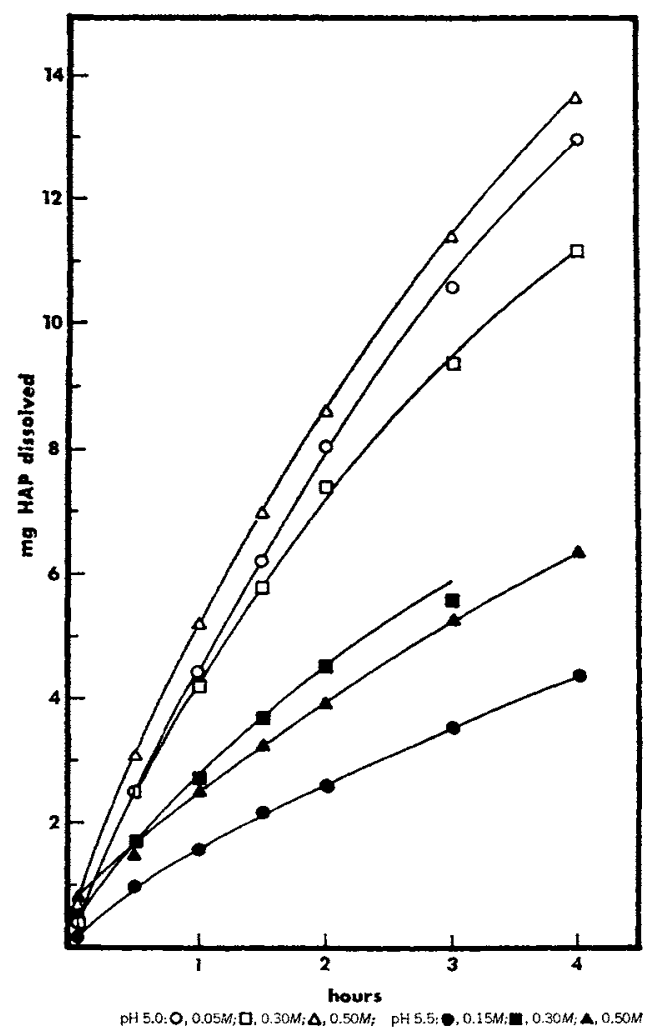

FIG 12.-Dissolution of TVA apatite using the disk method in $\mathrm{pH} 5.0$ and 5.5 acetate buffers and total ionic strength $=0.5 M$.

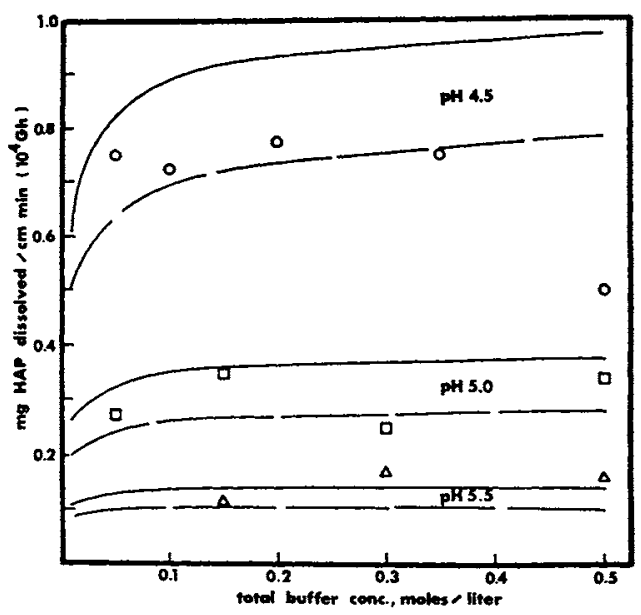

FIg 13.- Initial dissolution rates of TVA apatite using the disk method in acetic acid buffers. Smooth curves correspond to theory with $K_{\text {HAP }}$ $=1.0 \times 10^{-129}$. Broken curves correspond to theory with $K_{\mathrm{HAP}} 1.0 \times 10^{-131}$. The three sets of points are the experimental values at different $\mathrm{pH}$ conditions.

synthetic apatites and with powdered enamel. The initial dissolution rates were determined as a function of buffer concentration, $\mathrm{pH}$, and common ion concentration in acetic, lactic, and hydrochloric acid. The results of all these experiments were quantitatively compared to the model. 


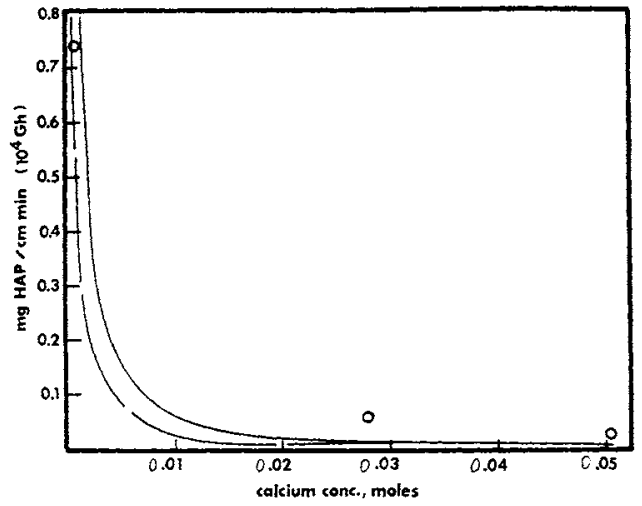

FIG 14.-Effect of the calcium common ion on the initial dissolution rates of TVA apatite using the disk method in $\mathrm{pH} 4.5$ and $0.05 \mathrm{M}$ total acetate-buffer concentration. The smooth curve corresponds to theory with $K_{\mathrm{H}_{A \mathrm{P}}}=1.0 \times$ $10^{-129}$, and the broken curve corresponds to theory with $K_{H: H}=1.0 \times 10^{-131}$. The circles are experimental values.

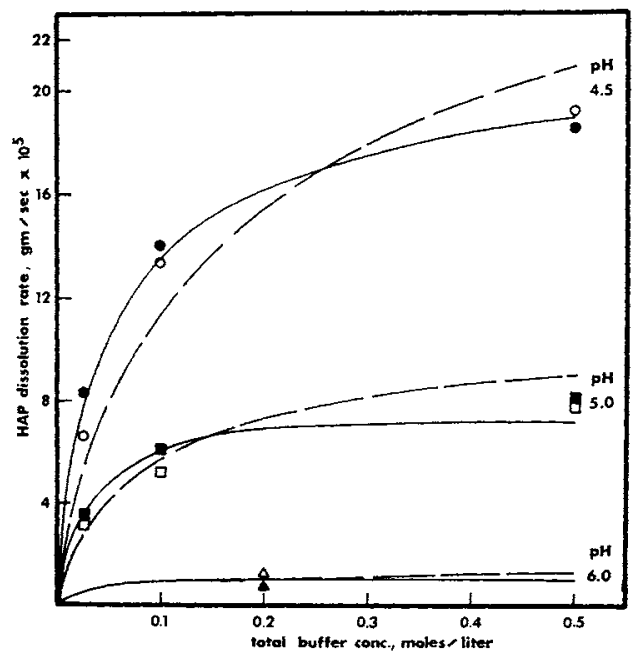

Frg 15.-Initial dissolution rates of Victor apatite in acetic acid buffers. Smooth curves correspond to theory when $K_{\mathrm{HAP}}=1.0 \times 10^{-115}$ and $k=1.34 \times 10^{4}$. Broken curves correspond to theory with $K_{\mathrm{HAP}}=1.0 \times 10^{-108}$ and $k=5.8 \times$ $10^{3}$. The three sets of symbols are the experimental values at different $\mathrm{pH}$ conditions. The closed symbols in each case correspond to the presence of $\mathrm{NaCl}$ in the buffers, and open symbols correspond to the absence of $\mathrm{NaCl}$.

It was found that all the experimental data with powdered enamel could be consistently described quantitatively by the hydroxyapatite model employing a single value

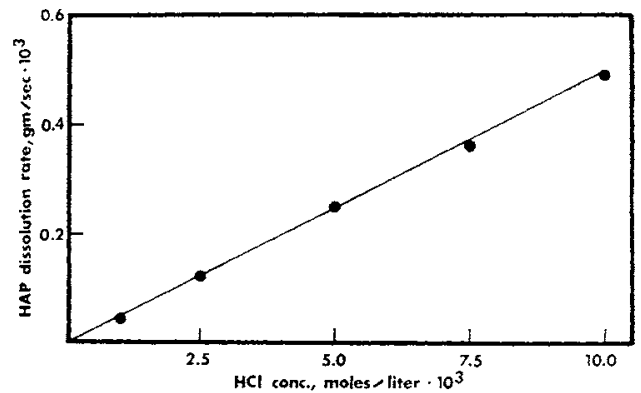

FIG 16.-Initial dissolution rates of enamel powder as a function of hydrochloric acid concentration in $0.5 \mathrm{M} \mathrm{NaCl}$.

for the solubility product. Particularly notable was the fact that these experimental data agreed with the model using the same solubility product value for hydroxyapatite that was quantitatively consistent with the data obtained by Gray on block-enamel dissolution rates.'

Experimental data obtained with a wellcrystallized synthetic hydroxyapatite sample (TVA) also agreed very well with the hydroxyapatite model with a single value for the solubility product. Both a powder method and a disk method were used in the experimental studies with this synthetic apatite sample.

This work has shown that the proposed hydroxyapatite model for the dissolution rate of enamel in acidic media is a very generally applicable one. It therefore should provide the basis for future investigations of more complex situations related to the mechanism of dental caries and oral biology and pathology in general.

\section{References}

1. Higuchi, W.I; Gray, J.A.: HefFerren, J.J.; and PATEL, P.R.: Mechanisms of Enamel Dissolution in Acid Buffers, $J$ Dent Res 44:330-341, 1965.

2. GrAY, J.A.: Kinetics of the Dissolution of Human Dental Enamel in Acid, J Dent Res 41:633-645, 1962.

3. Higuchi, W.I.; Parrot, E.L.; Wurster, D.E.; and HrGUCHI, T.: Investigation of Drug Release from Solids. II, $J$ Amer Pharm Assn Sci Ed 47:376-383, 1958.

4. Crank, J.: The Mathematics of Diffusion, Oxford: Clarendon Press, 1957.

5. TENNESSEE VALLEY AUTHORITY: Report No: 678, Alabama: Wilson Dam, Nov 6, 1956. 
6. Hefferren, J.J., and Higuchi, W.I.: Preparation of Large Quantities of Powdered Enamel, J Dent Res 44:455, 1965.

7. Manly, R.S., and Hodge, H.C.: Density and Refractive Index of Dental Hard Tissues, J Dent Res 18:133, 1939.

8. Patel, P.R.: Studies on Acid Demineralization Kinetics of Enamel, PhD thesis, University of Michigan, 1965.

9. GeE, A.; Domnngues, L.P.; and DeItz, V.R.: Determination of Inorganic Constituents in Sucrose Solutions, Anal Chem 26: 1487-1491, 1954.
10. Herrero-Lancina, M., and West, T.S.: Sensitive and Selective Spectrophotometric Reaction for Determination of Trace Amounts of Calcium, Anal Chem 35:2131$2135,1963$.

11. Becker, J.W.: Studies of a Proposed Model for Acid Demineralization of Hydroxyapatite, PhD thesis, University of Michigan, 1967.

12. Vinograd, J.R., and McBain, J.W.: Diffusion of Electrolytes and of the Ions in Their Mixtures, $J$ Amer Chem Soc 63: 2008-2015, 1941. 